\title{
Unprecedented accurate abundances: signatures of other Earths?
}

\author{
Jorge Meléndez ${ }^{1}$, Martin Asplund ${ }^{2}$, Bengt Gustafsson ${ }^{3}$, David Yong ${ }^{4}$ \\ and Iván Ramírez ${ }^{2}$ \\ ${ }^{1}$ Centro de Astrofísica da Universidade do Porto, Rua das Estrelas, 4150-762 Porto, Portugal \\ email: jorge@astro.up.pt \\ ${ }^{2}$ Max-Planck-Institut für Astrophysik, Germany \\ ${ }^{3}$ Institutionen för fysik och astronomi, Uppsala universitet, Sweden \\ ${ }^{4}$ Research School of Astronomy \& Astrophysics, Australian National University, Australia
}

\begin{abstract}
For more than 140 years the chemical composition of our Sun has been considered typical of solar-type stars. Our highly differential elemental abundance analysis of unprecedented accuracy $(\sim 0.01 \mathrm{dex})$ of the Sun relative to solar twins, shows that the Sun has a peculiar chemical composition with a $\approx 20 \%$ depletion of refractory elements relative to the volatile elements in comparison with solar twins. The abundance differences correlate strongly with the condensation temperatures of the elements. A similar study of solar analogs from planet surveys shows that this peculiarity also holds in comparisons with solar analogs known to have close-in giant planets while the majority of solar analogs without detected giant planets show the solar abundance pattern. The peculiarities in the solar chemical composition can be explained as signatures of the formation of terrestrial planets like our own Earth.
\end{abstract}

Keywords. Sun: abundances, Sun: fundamental parameters, solar system: formation, stars: abundances, stars: fundamental parameters, planetary systems: formation, Galaxy: abundances

\section{Introduction}

For many years people have wondered about how our Sun compares to other stars and to whether our existence is related to special properties of our solar system. Angelo Secchi compared the Sun to many bright stars (Secchi 1868). He classified the stars in three types: type I which is the modern class A and early F, type II which are M stars, and type III comprising modern class G, K and early F, and called by Secchi tipo solare (solar type), due to their spectroscopic similarity to the Sun. Furthermore, he concluded that "le stelle di questo terzo tipo mostrano di avere una composizione identica a quella del nostro Sole" (Secchi 1868), meaning that solar type stars have identical composition to our Sun. Further works (e.g. Payne 1925; Edvardsson et al. 1993; Reddy et al. 2003) have not conclusively shown whether the Sun has a normal composition or not, due to the relatively large ( $\gtrsim 0.05)$ remaining systematic errors (Gustafsson 2008). Thus, for more than 140 years father Secchi's conclusion on the "universal" solar composition of the Sun has remained valid. In order to make further progress, it is important to eliminate many of the systematic errors ( 0.05-0.1 dex) that plague stellar chemical composition analyses (Asplund 2005). Solar analogs, which are G0-G5 dwarfs, and solar twins, stars almost identical to the Sun (Cayrel de Strobel 1996), are important in this context, in particular solar twins, because due to their similarity to the Sun a highly differential analysis will cancel most systematic errors.

Thus, the first step in accurate comparisons of the Sun to other stars is to find solar twins. After many years of intensive search (see review by Cayrel de Strobel 1996), Porto 
de Mello \& da Silva (1997) found the first solar twin, 18 Sco [HD 146233], which is much closer to the Sun than previous candidates like 16 Cyg B [HD 186427] (see e.g. Fig. 2 of Meléndez et al. 2006). More recent surveys have largely increased the number of solar twins in the field (Meléndez et al. 2006; Meléndez \& Ramírez 2007; Takeda et al. 2007; Meléndez et al. 2009; Ramírez et al. 2009) as well as in the open cluster M67 (Pasquini et al. 2008). The most productive survey at high resolution $(\mathrm{R} \sim 60,000-110,000)$ is being undertaken by our group. Most Northern solar twin candidates were observed with the $2.7 \mathrm{~m}$ telescope at the McDonald observatory, and complemented with data obtained at the Keck observatory. The Southern targets were observed using the Magellan Clay $6.5 \mathrm{~m}$ telescope at Las Campanas, complemented with recent (August 2009) VLT observations.

Our solar twin project started in 2002, when only one solar twin was known. In order to improve our chances of finding solar twins, we first expanded the color-temperature relations of Alonso et al. (1996) to other photometric systems (Meléndez \& Ramírez 2003), allowing us to use existing photometry in other systems (e.g. Geneva) to select the best targets. We later improved the calibrations adding more stars and including new homogeneous systems like Tycho $\left(B_{T}-V_{T}\right)$ and 2MASS (Ramírez \& Meléndez 2005). Although our first solar twin proposal in 2004 did not fly, the same year a more "exciting" proposal on $\mathrm{Li}$ in halo stars was granted a few nights with Keck. A small amount of that observing time was devoted to twin candidates, resulting in the discovery of the second solar twin (HD 98618; Meléndez et al. 2006) and revealing that our temperature scale (and that of Alonso et al. 1996), although precise, have probably a zero-point issue. Our new selection of candidates from the Hipparcos catalog took into account a preliminary zero-point offset, which has been recently confirmed by analyses of solar twins and used for improved temperature calibrations (Casagrande et al. 2009).

New solar twin proposals at McDonald and Magellan at Las Campanas (through Australian access) during 2006 were successful. The first observing run at McDonald in April 2007 allowed us to identify the best solar twin known to date, HIP 56948 (Meléndez \& Ramírez 2007), which is not only very similar to the Sun physically, but has also a low Li abundance similar to solar. Its status of best solar twin has been recently confirmed by Takeda \& Tajitsu (2009). On the other hand, the Magellan observations at Las Campanas are opening new windows for astrophysics of the 0.01 dex level in chemical abundance accuracy. New observations taken recently at the VLT with UVES and CRIRES, promise to achieve even better precision $(0.005 \mathrm{dex}, \sim 1 \%)$, and to use solar twins to set tight constraints on Li and Be depletion in the Sun (e.g. do Nascimento et al. 2009).

\section{Terrestrial planet signatures}

Our observations taken with the MIKE spectrograph and the $6.5 \mathrm{~m}$ Magellan Clay telescope at Las Campanas, have definitely shown, for the first time, that the Sun has a peculiar chemical composition (Meléndez et al. 2009). As can be seen in Fig. 1, the difference in chemical abundances between the Sun and the mean value of the solar twins does not cluster around zero. Instead, the abundance differences have a remarkable correlation with the condensation temperature of the elements (Lodders 2003). This peculiarity has been recently verified by Ramírez et al. (2009) using McDonald observations.

A fascinating possibility to explain the chemical anomalies of the Sun is that they are related to its properties as a host of terrestrial planets. A particularly striking circumstance is that the inner solar system planets and meteorites are enriched in refractories compared to volatiles (e.g. Alexander et al. 2001 ), with a break at $T_{\text {cond }} \sim 1200 \mathrm{~K}$, identical to the break at $T_{\text {cond }} \sim 1200-1250 \mathrm{~K}$ detected in the solar abundance pattern (Fig. 1 ). The abundance pattern of meteorites is a mirror-image of the solar pattern relative to 


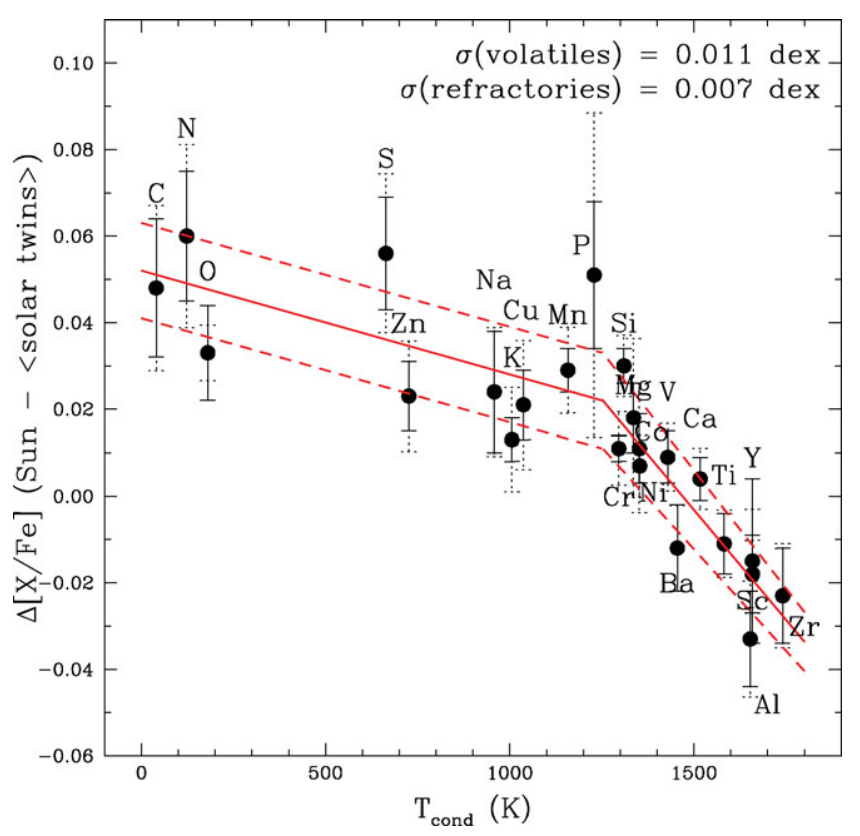

Figure 1. Differences between $[\mathrm{X} / \mathrm{Fe}]$ of the Sun and the mean values in the solar twins as a function of $T_{\text {cond }}$. The abundance pattern shows a break at $T_{\text {cond }} \sim 1200-1250 \mathrm{~K}$. The solid lines are fits to the abundance pattern, while the dashed lines represent the standard deviation from the fits. The low element-to-element scatter from the fits for the refractory $(\sigma=0.007 \mathrm{dex})$ and volatile $(\sigma=0.011 \mathrm{dex})$ elements confirms the high precision of our work. Observational errors (including errors in both the Sun and solar twins) are shown with dotted error bars, while the errors in the mean abundance of the solar twins are shown with solid error bars.

the solar twins. Also, a radial gradient exists, with greater enhancement of refractories at smaller heliocentric distances (Palme 2000). The break in the chemical abundance pattern at $T_{\text {cond }} \approx 1200 \mathrm{~K}$ (Fig. 1 ), suggests that the volatiles retained their original abundances both in the Sun and the solar twins. Such temperatures are only encountered in the inner parts ( $<3 \mathrm{AU}$ ) of proto-planetary disks (Ciesla 2008), which also suggests that the abundance pattern is related to the presence of terrestrial planets. Furthermore, iron meteorites (like those we see in museums around the world) were probably formed in the terrestrial planet region and may be a remnant of Earth forming material (Bottke et al. 2006), hence being perhaps formed from the iron missing in the Sun.

The amount of dust-depleted gas required to explain the solar abundances is similar to the mass of refractories locked up in Mercury, Venus, Earth and Mars (Meléndez et al. 2009). Thus, it is tempting to speculate that the formation of the terrestrial planets might have given the Sun its special surface composition. The disk masses during the T Tauri phase are $\sim 0.02 M_{\odot}$ but larger values are possible, providing thus enough material to change the solar photospheric composition. However it may be a problem with time-scales since proto-planetary disks are observed to have typical life-times $<10 \mathrm{Myr}$ (Sicilia-Aguilar et al. 2006), but the deep solar convection zone at that time would have erased the planetary signatures, unless the proto-solar nebula was abnormally long lived so that the dust-depleted gas was accreted $\sim 20$ Myr later when the solar convection zone was thin. Another possibility is that the early Sun was never fully convective, as shown by the dynamical star-formation calculations with a time-dependent convection treatment of Wuchterl \& Tscharnuter (2003) and Wuchterl \& Klessen (2001), making it easier to imprint the signatures of other Earths (Nordlund 2009). 
We have also analyzed a sample of 10 solar analogs from planet surveys, four having inner giant planets, while for the other six no planets have been detected yet. Our analysis shows that all solar analogs with giant planets differ from the Sun but closely resemble most solar twins. Thus, the odd solar composition is not due to giant planets as such, but probably related to terrestrial planets. Indeed, only two of the six solar analogs without close-in giant planets have abundances that differ significantly from the solar pattern. The fraction of stars with the solar pattern seems thus tentatively related to the presence of giant planets on close orbits: $\approx 0 \%$ when having such planets and $\approx 10-20 \%$ for solar-type stars in general, and $\sim 50 \%-70 \%$ without close-in giant planets. Although the statistics has to be improved considerably, the numbers are clearly tantalizing.

\section{How typical is our solar system?}

Although the samples are still relatively small, considering both the Magellan (Meléndez et al. 2009) and McDonald (Ramírez et al. 2009) samples, we conclude that about $10-20 \%$ of stars physically similar to the Sun have also chemical similarities to our Sun and therefore they may be hosting terrestrial planets like our own Earth.

A complete high precision spectroscopic survey of solar twins is urgently needed in order to know which stars may be the best candidates of hosting solar system twins and to improve the statistics on the fraction of stars hosting other Earths and perhaps life.

\section{References}

Alexander, C. M. O'D., Boss, A. P., \& Carlson, R. W. 2001, Science, 293, 64

Alonso, A., Arribas, S., \& Martinez-Roger, C. 1996, A\&A, 313, 873

Asplund, M. 2005, ARAA, 43, 481

Bottke, W. F., Nesvorný, D., Grimm, R. E. et al. 2006, Nature, 439, 821

Casagrande, L., Ramírez, I., Meléndez, J., Bessell, M., \& Asplund, M. 2009, A\&\&A, submitted

Cayrel de Strobel, G. 1996, A\& AR., 7, 243

Ciesla, F. J. 2008, Meteoritics Planet. Sci., 43, 639

Do Nascimento, J. D., Jr., Castro, M., Meléndez, J. et al. 2009, A\&SA, 501, 687

Edvardsson, B., Andersen, J., Gustafsson, B. et al. 1993, A\&BA, 275, 101

Gustafsson, B. 2008, Physica Scripta, T130, 014036.1

Lodders, K . 2003, ApJ, 591, 1220

Meléndez, J., Dodds-Eden, K., \& Robles, J. A. 2006b, ApJ (Letters), 641, L133

Meléndez, J. \& Ramírez, I. 2007, ApJ (Letters), 669, L89

Meléndez, J., Asplund, M., Gustafsson, B., Yong, D. 2009, ApJ (Letters), 704, L66

Nordlund, A. 2009, ApJ (Letters), submitted, arXiv:0908.3479

Palme, H. 2000, Space Sci. Rev., 92, 237

Pasquini, L., Biazzo, K., Bonifacio, P., Randich, S., \& Bedin, L. R. 2008, A\&GA, 489, 677

Payne, C. H. 1925, Ph.D. Thesis

Porto de Mello, G. F., \& da Silva, L. 1997, ApJ (Letters), 482, L89

Ramírez, I., \& Meléndez, J. 2005, ApJ, 626, 465

Ramírez, I., Meléndez, J., \& Asplund, M 2009, A\&BA (Letters), submitted

Reddy, B.E., Tomkin, J., Lambert, D. L., \& Allende Prieto, C. 2003, MNRAS, 340, 304

Secchi, A. 1868, Sugli spettri prismatici delle stelle fisse, Roma : Tipografia delle belle arti

Sicilia-Aguilar, A., Hartman, L. W., Füréz, G., et al. 2006, AJ, 132, 2135

Takeda, Y., Kawanomoto, S., Honda, S., Ando, H., \& Sakurai, T. 2007, A $\& A$ A, 468, 663

Takeda, Y. \& Tajitsu, A. 2009, PASJ, 61, 471

Wuchterl, G. \& Klessen, R. S. 2001, ApJ, 560, L185

Wuchterl, G. \& Tscharnuter, W. M. 2003, A\& $A, 398,1081$ 